\title{
Two-Dimensional Medical Image 3D Visualization System's Realization
}

\author{
Ai Ting ${ }^{1}$, Li Zhe ${ }^{1}$, Miao Yu ${ }^{2}$ \\ ${ }^{1}$ Engineering and Technology College of Changchun Department of Electronics \\ ${ }^{2}$ Science and Technology University of Changchun Department of computer \\ Email: aiting121@sina.com
}

Received 2012

\begin{abstract}
With the development of virtual reality application in the medical field, two-dimensional medical image of the three-dimensional visualization technology made possible. Surgery gets into minimally invasive operation microscopy Era, and gradually becomes a new research hotspot. This paper studies the realization of two-dimensional medical image 3D reconstruction visualization system method, and the overall process and management module. Using the main technology of VTK (The Visualization Toolkit) to achieve a two-dimensional medical image three-dimensional visualization system, which can help the physician to obtain help clinical diagnosis Information and play an important role in treatment, accurate positioning in diseased tissue and tumor early diagnosis.
\end{abstract}

Keywords: VTK; Visualization in Scientific Computing; 3D Reconstruction; Virtual Endoscopy

\section{Introduction}

In the medical field, virtual reality technology and modern surgical operation combining the virtual operation system for surgical operation, operation guidance and assessment of teaching and provides a new mode. It gradually shows its great potential and prospects. With the development of medical imaging technology, Virtual operation scene creation is the main organs of the human body $3 \mathrm{D}$ modeling, computer tomography $(\mathrm{CT})$, magnetic resonance imaging (MRI) and other devices to obtain patient two-dimensional image information for 3D reconstruction, direct generation for virtual operation system geometric model so that the image can intuitively show the internal body tissues with complex structures, help doctors diagnosis and guide the operation, the auxiliary means can make up the deficiency in imaging equipment, can provide users with a realistic 3D medical image, which is convenient for doctors from much angle, much level were observed and analyzed, and could allow doctors to participate effectively in data processing and analysis.

\section{Medical Image Visualization Technology}

Medical image visualization is the transformation from CT, MRI and other digital imaging technology to obtain the information of human body visually on the computer performance for the three-dimensional effect, so as to provide with tradition al method to obtain structural information. In the medicine field, the information of human body is obtained by CT, MRI and other digital im- aging technology which to be visually on the computer performance for the $3 \mathrm{D}$ effect. $\mathrm{X}$ ray tomography image $\mathrm{CT}$ and magnetic resonance images MRI in clinical application will produces a large amount of data such as Figure 1, which can effectively display the information contained in these data, and make full use of these data, which has a wide range of applications and research value in the medical field.

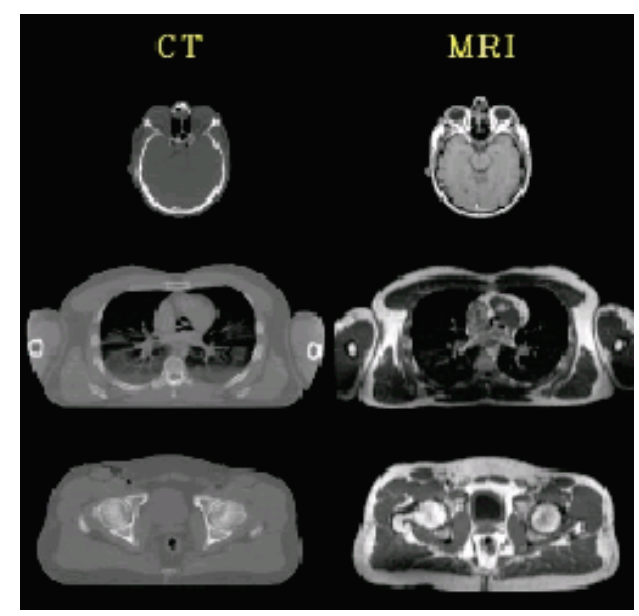

Figure 1. medical image data.

\subsection{D Visualization of Spatial Data Process}

There are a lot of methods about the image of the 3D visualization, but the basic steps are roughly the same, as 
shown in Figure 2. First of all, we will input the two-dimensional images to computer, which convenient processing format. Through a two-dimensional filter, we can reduce the image noise effects, improving the signal to noise ratio and the elimination of image's wake. Take the medicine image interpolation method, the key parts of isotropic processing, access to data ${ }^{[1]}$. After three filters, different tissues and organs require segmentation and classification, on the same site of the different image registration and fusion, in order to facilitate further to a region of interest of the operation. According to the different requirements of the $3 \mathrm{D}$ visualization and system capacity, choose a different method for 3D rendering, 3D reconstruction.

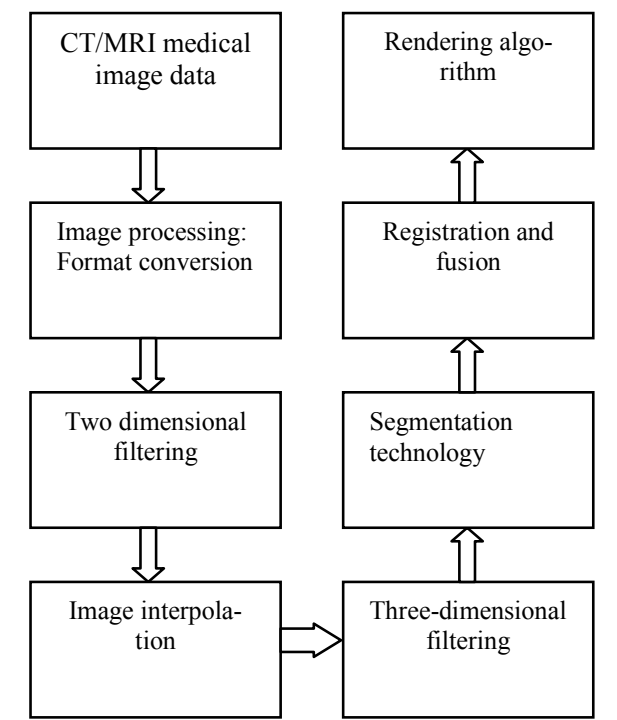

Fig 2 the medical image 3D visualization steps

\subsection{Image visualization algorithm}

Three-dimensional visualization technology is usually divided into surface rendering and volume rendering of two methods for.

1) Surface rendering

The method of surface rendering is that, first structure out of the middle of geometric primitives by the $3 \mathrm{D}$ data (such as surface, plane etc.) and then by the traditional computer graphics techniques such as reasonable illumination model, texture mapping method to achieve the picture drawing ${ }^{[2]}$. Surface rendering is the most famous of the marching cubes algorithm (Marching, Cubes, $\mathrm{MC})$.

2) Volume rendering

Volume rendering is the method to process the volume data field in each element, namely the voxel. Voxel is the basic unit in three dimensional, which is composed of two pieces of adjacent sections of each of the four points of a cube ${ }^{[3]}$. They are generally considered to have certain properties (opacity and brightness etc), through the calculation of all voxels on the light, at the same time interactively adjust opacity, light effects and other parameters, and then synthesized with three-dimensional effect of the image, and direct the 3-D gray-scale data projection display onto a two-dimensional screen, so the calculation a large quantity, image generation is slower, more by hardware technology development limited, more difficult to implement than the surface rendering.

The difference of Volume rendering and the tradition of surface rendering are shown in figure 3. From the image quality, volume rendering is better than surface rendering. But from the interactive performance and computational efficiency, at least in the current hardware platform, surface rendering is superior to volume rendering, because the surface rendering is the traditional graphics rendering algorithm, the existing interactive algorithm and graphics hardware and graphics technology can fully play a role ${ }^{[4]}$.

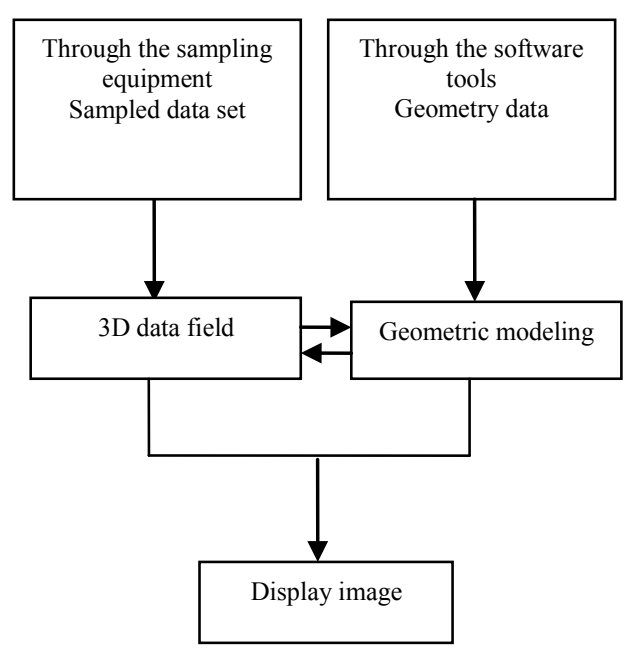

Fig3. The comparison of two kinds of drawing method

\section{Reconstruction of 3D Visualization System's Realization}

\subsection{VTK's Basic function}

The main function of VTK is for image processing, computer graphics and visualization in scientific computing, especially for three-dimensional reconstruction of functions (such as surface rendering and volume rendering ability and powerful image processing capability) ${ }^{[5]}$. VTK graphics and visualization in the field of commonly used algorithm package into a class, users in visual development process does not need to consider the specific details, which can bring great convenience into research and development.

The process of VTK visualization is use the pipelining mechanism to achieve. That is to say in the visualization process, above result as an input of next process. Frame structure as shown in figure 4. 


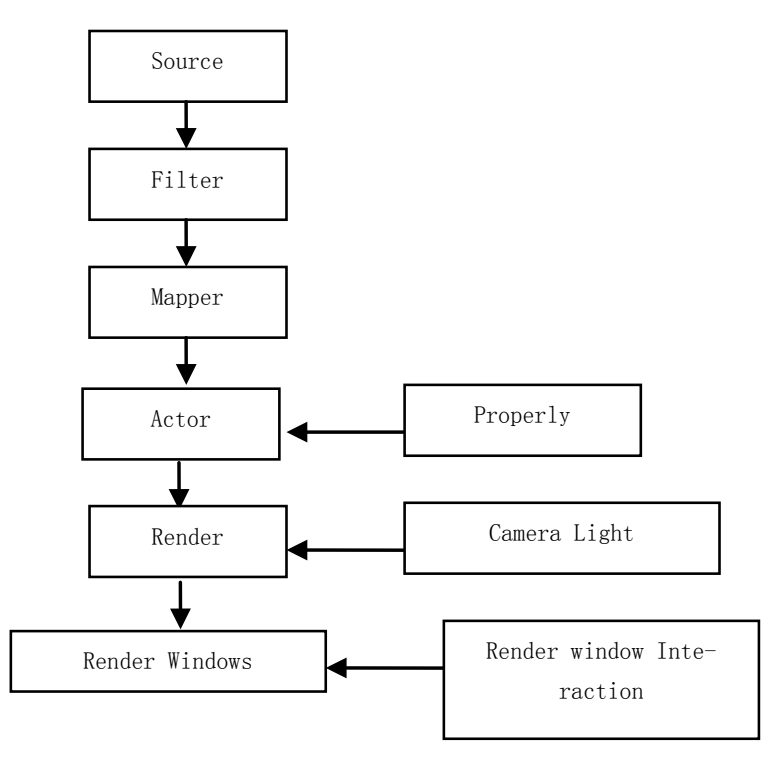

Fig 4 the VTK object model framework

First of all, we can establish a data line (Data pipeline) to process data and link up the Sources Filters and Mappers; secondly, establish the appropriate target graphics to show data.

The system used VTK as the basic architecture and design the data flow pipeline mode. This design uses B/S structure, in addition to install some necessary plug-in, does not need to be installed in the client of other complex software. The system includes the following modules: the role management module, including hospitals, doctors, patients, experts, the system administrator; data module, including the role of books management as well as the original medical image is processed and data management; data visualization module, mainly DICOM Web data visualization and processing results of Web three-dimensional visualization ${ }^{[6]}$.

The scalability must be considered in module design fully. Several modules must independent design and code: DICOM data read module, tissue segmentation module, cutting management module, virtual endoscope mirror module, 3D reconstruction module, pseudo color management module, management module, three view section frame management module ${ }^{[7]}$.

$3 \mathrm{D}$ reconstruction in the whole system is at the core position in management. Module to call the relationship between such as shown in Figure 5 below:

\subsection{System's Realization}

1) Development environment

The system is development language for the $\mathrm{VC}++$, in which use the visualization toolkit VTK.

2) The technology of virtual endoscope
Reconstruction these two-dimensional tomographic images which obtained through CT and MRI, can show

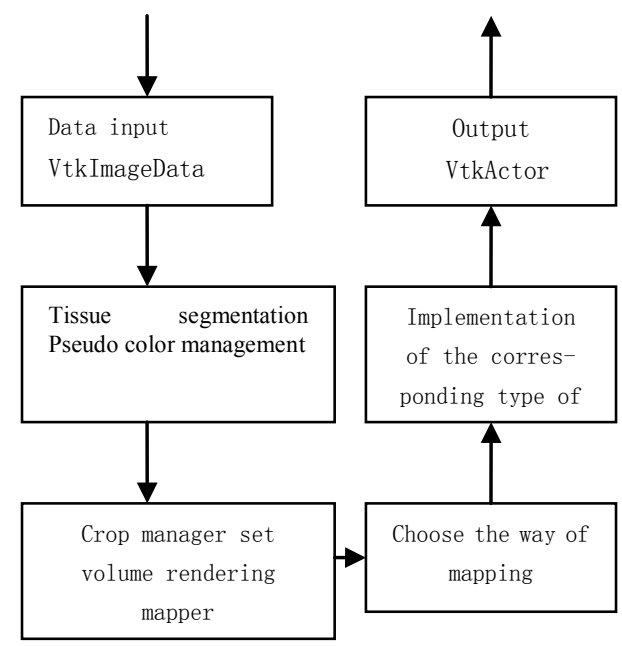

Fig 5 3D reconstruction data flow diagram

the interior of the body structure ${ }^{[8]}$. Then the virtual reality and virtual endoscopic technique, the doctor through the mouse to perform various operations, such as: translational, rotational, parameter setting, in a three-dimensional internal cavity roaming, simulation of the traditional endoscope a variety of behavior. Figure 6 is the virtual endoscope display of human kidney organ three-dimensional images (a, B, C, D).

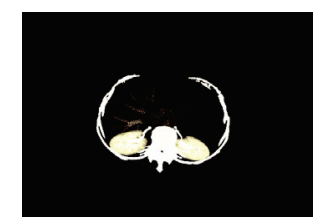

a. Input image

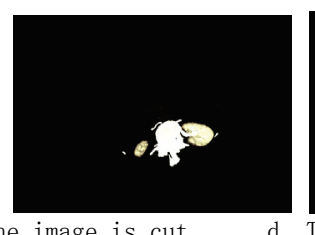

c. The image is cut
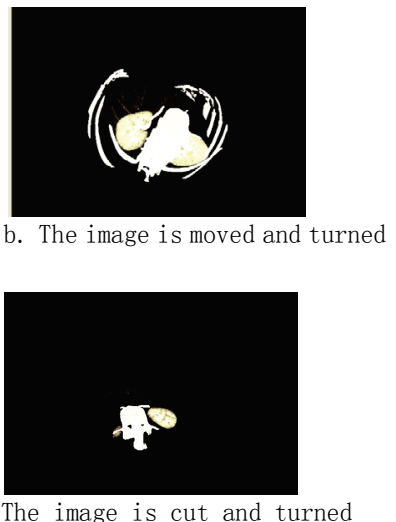

Fig 6. the virtual endoscope display 3D images of human kidney

1) Navigation and interaction

The existing navigation mainly has the following several methods: manual navigation; users adjust to the camera directly; through a mouse sliding lens user can see the inner surface anything, which give user the most freedom. Guidance navigation that can accord user defined path to adjust the camera and convenient effect reproduction. It is drawn in a path specific scene, gives an intuitive representation. Automatic navigation, which 
automatic path generation, automatically generates a navigation path; mark the inner wall of the pipe center line and fast show paths ${ }^{[9]}$.

In this system, it mainly realized in front of the two navigation way. For the third navigation way, we need to study the removal of impurities, multiple branch path smooth, key technology, which is a very promising method of navigation and be researching in further.

After loading, the CT pictures displayed on the computer screen, as shown in figure 7.a and figure 7.b.Can be found by clicking the "lens" button to move the image, from different angles to observe the structure, whether the incidence of lesions. Click the "cutting" buttons, the image cutting, and then through the magnification of the image were observed, helping to make the correct diagnosis.

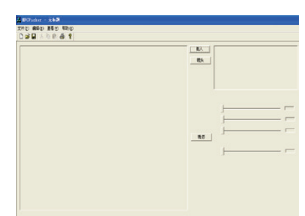

Fig 7. a system interface

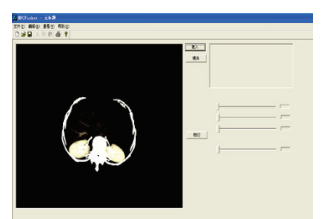

Fig 7.b CT image loading.

\section{Conclusion}

These articles realize two-dimensional images of three-dimensional visualization system which set out from the $3 \mathrm{D}$ visualization system construction method and module management two respects. The realization of the system integrated the information of many aspects. It is the combination of design about the virtual reality technology and modern medicine ${ }^{[10]}$. And it is the multidisciplinary research. The system is capable of using multiple imaging or multiple imaging devices for information, to provide the defects which caused by the data information is not accurate or uncertainty ${ }^{[10]}$.

It can be more comprehensive and accurate in clinical diagnosis, treatment, radiotherapy, plan design, surgical operation and curative effect evaluation.

\section{REFERENCES}

[1] M Haubner, C Krapichler, A Losch, et al. Virtual reality in medicine- computer graphics and interaction techniques[J]. IEEE Trans.On Information Techology in Biomedicine, 1997, 1(1):61-72.

[2] Hamann, B.A data reduction scheme for triangulated surface $[\mathrm{J}]$. Computer Aided Geometric Design, 1994,11(2):197-214.

[3] Craig Upson, Michael Keeler, V-buffer. Visible volume rendering $[\mathrm{J}]$. ACM SIGGRAPH Computer Graphics, 1988, 22(4): 59-64.

[4] J Bloomenthal. Polygonalization of Implicit Surfaces[J]. CAGD, 5(4):341-355, 1988.

[5] Ghrare S E,Ali M A M,Jumari K,et.An efficient low complexity lossless coding algorithm for medical images[J].American Journal of Applied Sciences, 2009,6(8):1502-1508

[6] Kelly, P.J. (1994). Quantitative Virtual Reality Surgical Simulation, Minimally Invasive Stereotactic Neurosurgery and Frameless Stereotactic Technologies. Medicine Meets Virtual Reality II: Interactive Technology \& Healthcare: Visionary Applications for Simulation Visualization Robotics (pp. 103-108).

[7] Selvaraj K.Data extraction from computer acquired images of a given $3 \mathrm{D}$ environment for enhanced computer vision and its applications inkinematic design of robs[J].Journal of Computer Science,2010,6(4):425-427.

[8] Purschke F,et al.Virtual Reality:New Methods for Improving and Accelerating the Development Process in Vehicle Styling and Design.Computer Graphics International,Proceedings, 1998.789.

[9] Yang Mengzhou,Shi Jiaoying.3D Model Reconstruction Algorithm Based on the Texture of Photos[J].Ruan Jian Xue Bao/Journal of Software,2000,11(4):502-506.

[10] Xu B, et al. Video Metrics and Optical Methods for 3D Shape Measurement,Proceedings of SPIE[J].the International Society for Optical Engineering,2001,4309:26-33. 